\title{
Progress of Applied Seismo-Electromagnetism
}

\author{
Yukio Fujinawa1, Yoichi Noda² \\ ${ }^{1}$ Organization for Development of Resilient Community, Chiyoda-ku Tokyo, Japan \\ ${ }^{2}$ Institute of Oceanic Research and Development, Tokai University, Shimizu-ku, Shizuoka, Japan \\ Email:hjfujinawa@cl.cilas.net
}

How to cite this paper: Fujinawa, Y. and Noda, Y. (2020) Progress of Applied Seismo-Electromagnetism. Open Journal of Earthquake Research, 9, 1-18. https://doi.org/10.4236/ojer.2020.91001

Received: November 18, 2019

Accepted: January 4, 2020

Published: January 7, 2020

Copyright $\odot 2020$ by author(s) and Scientific Research Publishing Inc. This work is licensed under the Creative Commons Attribution International License (CC BY 4.0).

http://creativecommons.org/licenses/by/4.0/ (c) (i) Open Access

\begin{abstract}
It is a natural process of the development of technology that isolated findings are synthesized for the solution of problems for each country. We previously introduced an idea to solve one of serious global problems, i.e. energy consumption and risks threating present and future mankind. The Resilient Smart City is one of concepts in the re-adjustment of energy consumption, global warming and the increase of risks. It uses disaster mitigation information, especially the earthquake early warning (EEW) and earthquake predictions. As for EEW, Japan has had an extensive system since 2007, and has proved that the system works well enough to save considerable amount of human lives and important facilities at the times of several major earthquakes. Another tool is nothing but the earthquake prediction. Progress of seismo-electromagnetics can contribute to solving those needs. Here we present a summary of our works concerning earthquake prediction in these 30 years.
\end{abstract}

\section{Keywords}

Global Problem, Earthquake, Warning, Prediction, Electromagnetic Phenomena, Precursor

\section{Introduction}

There are many countries or regions suffered by strong earthquakes in the world as Japan, China, Indonesia, Turkey, Italia, USA, Central America, South Ameri$\mathrm{ca}$, and so on. One of the most efficient disaster mitigations has been assumed to predict them and take appropriate actions beforehand to save human lives before occurrence of the events. We have only one successful example of earthquake prediction, the Haicheng Earthquake (1975 Feb. 04) in China. Many countries, especially earthquake-prone countries have been involved to find practical methods more than half of a century. But there are still no practical methods despite great efforts. There spreads general pessimistic atmosphere 
against positive stance to the earthquake prediction with the result of decrease of number of scientists and engineers interested in the investigation. Notwithstanding, there remain still not a small number of experts engaged in the earthquake prediction and try to find a new breakthrough.

We have been investigating underground electric field anomalies since 1989 using special antenna. Analyses of the large amount of data at different geological sites and for crustal activities from 1989 to 2002 have revealed that there are plausible anomalies in both ULF and VLF bands. In addition, we found the electric signals propagated some ten times faster than the seismic P wave, i.e. 100 $\mathrm{km} / \mathrm{s}$, suggesting the possibility of much earlier alarms compared to EEW based on seismic waves. The most interesting and profitable evidence for the prediction was detected at the time of the great Tohoku earthquake in 2011. The project was planned to reveal the source of the high-speed phenomena propagating such a high speed several times of seismic P-wave by using measurement instrument of wider frequency band. The special pulse-like variations in the ELF band were found to occur only in the nucleation stage just a week before the earthquake, suggesting that the practical method of imminent prediction is possible. Analyses suggested that the events were caused by micro-cracks occurring in the nucleation stage, having features consistent with recent theoretical and experimental results in seismo-electromagnetism. Results of research in seismo-electromagnetics have a wide scope of applications in variety field of technology.

\section{Method}

\subsection{Sensor}

We use a special sensor, made of a long vertical steel casing pipe that is installed into a borehole $200 \mathrm{~m}$ to $1800 \mathrm{~m}$ deep. The antenna invented by [1] is of the mono-pole type and is immersed underground (Figure 1) in the conductive crust analogous to the towing tail cable antenna used for submarines underwater communication. The steel casing pipe functions as a monopole of the antenna in the conductive substance and the copper wire buried underground, surrounding the casing pipe, as the reflector [2] [3] [4] [5]. The boreholes were originally for different purposes such as for the measurements of seismic motion, ground water level and chemical contents, ground deformation, pumping of natural gas and belonged to several organizations (Fujinawa et al. [6] [7]). The recording frequencies are DC band $(0-0.7 \mathrm{~Hz}), \mathrm{ULF}(0.001-0.7 \mathrm{~Hz})$ and ELF/VLF $(1 \mathrm{kHz}$ - $9 \mathrm{kHz}$ ). The electromagnetic field observation stations have been constructed in central Japan since 1988, and thirteen observation sites were used (Figure 2).

\subsection{Observation}

Prior observation confirmed that the system has high robustness against both meteorological and urban noise, enabling us to find signals related to crustal activities [4] [5] [6] [7]. Exceptions are the large noises induced by nearby 


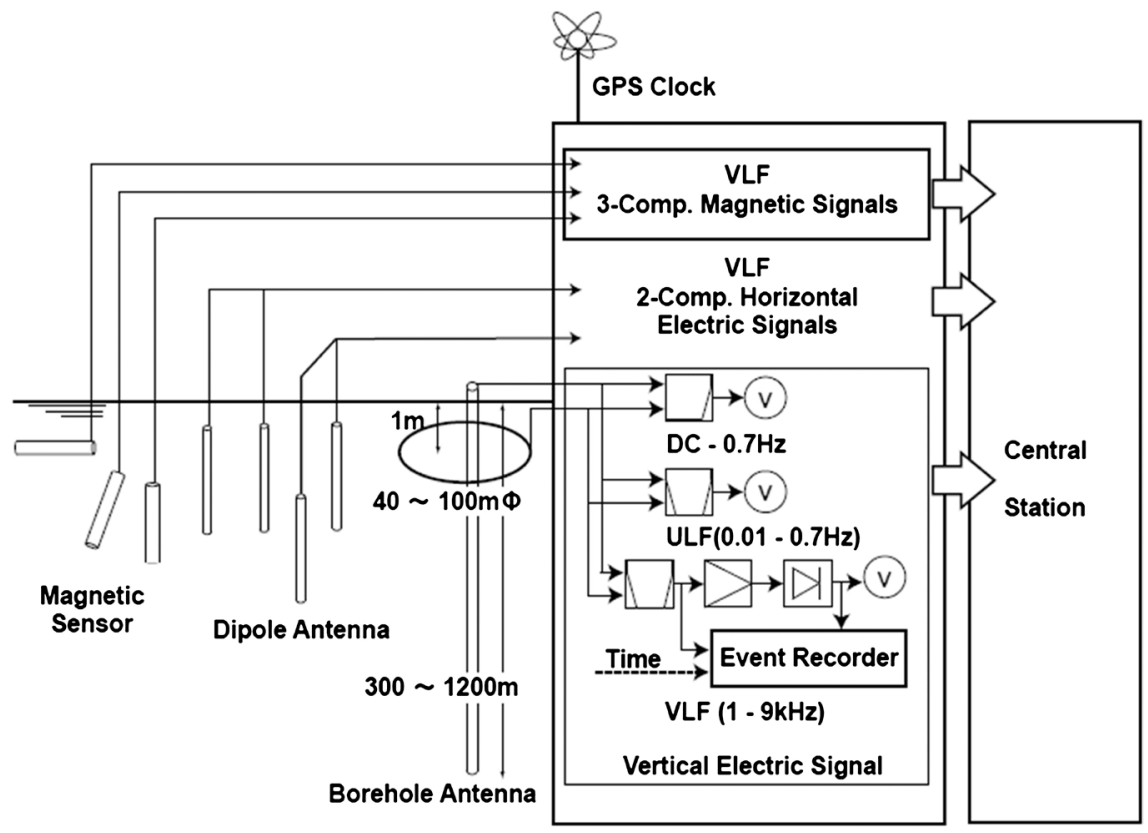

Figure 1. Schematic diagram of multiple component electromagnetic field observations. The electric field is measured by a pair of horizontal dipoles of $8 \mathrm{~m}$ in length with a span of $10 \sim 50 \mathrm{~m}$ and using a vertical mono-pole of $100-1200 \mathrm{~m}$ length surrounded by wire with a radius about $5-50 \mathrm{~m}$ buried at a depth of about $1 \mathrm{~m}$.

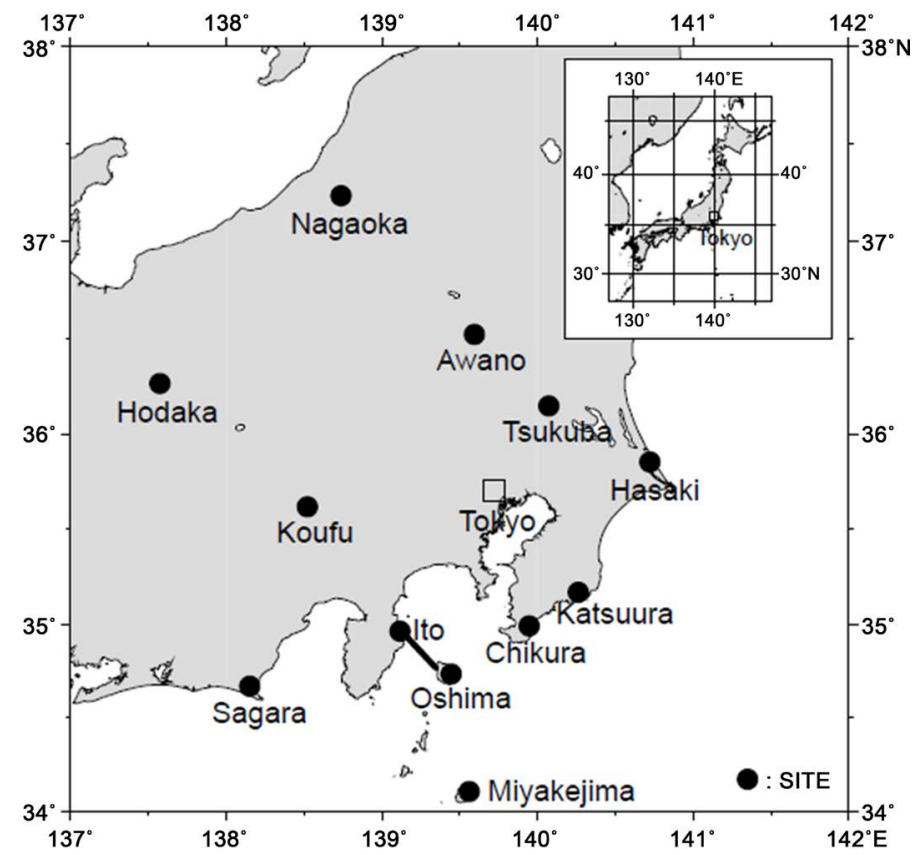

Figure 2. Locations of the electro-magnetic field observation sites in central Japan utilizing borehole antenna $150-1200 \mathrm{~m}$ long as the principal sensor at eleven sites: Hasaki (HAS), Katsuura (KTU), Tsukuba (TKB), Chikura (CKR), Awano (AWN), Izu-Oshima (OHS), Miyakejima (MIY), Nagaoka (NGO), Koufu (KOF), Sagara (SAG) and Hodaka (HOD).

lightning discharges, which are easily discriminated because of large difference of strength and waveform. After confirming the performance of the borehole 
antenna our observation was conducted only with the borehole antenna without magnetic sensors and electric dipoles (Figure 1).

The observation network was built and has operated since 1989, and the sites are situated at several kinds of geological conditions, which are nearly uniform in central Japan (Figure 2). Several kinds of anomalies were picked up and investigated from the points of view of finding precursors related to earthquakes and volcanic eruptions. The ULF band anomalies [3] have been most extensively investigated because they were detected in almost all cases of nearby volcanic eruptions and seismic swarms [5] [6] [7].

\subsection{Temporal Experiment}

Sometimes temporal experiments were focused on topics. For example, we temporally made high-speed recordings with a time resolution of $1 \mathrm{sec}$ for about 27 months (from June 1994 to December 2002) to investigate the characteristics of precursory anomalous signals. These experiments suggested that there are electromagnetic variations starting from the origin times of moderate earthquakes. Moreover, the evolution of signals correlated with seismic motion at the site, some $100 \mathrm{~km}$ distance from the epicenter.

We could not analyze higher-frequency phenomena in detail because of the system's slow recording speed. Our new observation, which started on March 3, 2011 at Hasaki (now Kamisu City, Ibaraki Prefecture) enabled us to investigate particular co-rupture signals using an updated dynamic range. We could detect very interesting electric variations, suggested to be induced by microcracks in the nucleation stage of earthquakes [8].

\section{ULF Band Anomalies}

\subsection{Observational Result}

Electromagnetic approaches have been providing many interesting results, shedding light on the preparatory process for earthquakes [9] [10] [11]. There are many observations of electromagnetic anomalies to be asserted as precursory phenomena. However, there are few well-organized observations that confirm intrinsic relationships between the crustal fracture process and the candidates of precursors. We need a framework of investigation containing generally approved evaluation criteria [12]. Here, we present the electric field observation from a sensitive special sensor referring to the guidelines as far as possible.

Moreover, there are many generation mechanisms that induce electromagnetic anomalies. The fluid-phase has been noted to understand the complex physio-chemical process of earthquakes. Fluids are suggested to play important roles in long-term structural evolution, fault creeping, and fault ruptures (e.g., [13] [14] [15]. Ground water levels and chemical content have been observed in quantities that suggest the existence of changes in the ground water regime before an earthquake (e.g., [9] [16] [17] [18]). A new generation model was presented to explain the characteristic features of the GUV [5] [6]. 


\subsection{Characteristics of Anomalies}

The ULF band characteristic waveform was first identified at the time of volcanic eruption activities in 1990 at Izu-Oshima [3]. The amplitude increased suddenly before gradual decreasing to about half the peak amplitude, then decreasing sharply to one-tenth of the peak, and finally returning to the gradual trend level (Figure 3). The signals have never been detected in a normal state, but have been observed in almost all volcanic eruption activities and seismic swarms occurring near the observation sites: the earthquake swarm around Mt. Hodaka in 1998 [7] and Mt. Nikko-Shirane in 1999, and the volcanic eruption at the Miyake Island in 2000. The phenomena have been suggested to be induced by rapid ground motions through the electro-kinetic effect [19] [20] [21] [22] [23]. The ULF variation is called: "geyser like ULF signals "(GUV) because of the similarity of the temporal change of the electric strength with the water height change of the geyser. We thought that the whole of the pulse-like variations detected by the slow speed recordings are of this type.

\subsection{Generation Mechanism}

Electromagnetic field variations, as self-potential change, have been attributed to piezo-effects, MHD effects, and electro-kinetic effects [19] [20]. Quantitative comparison of these effects suggests that the electro-kinetic effects are about one-order larger than other effects [24]. [22] first attempted to explain the magnetic anomalies associated with the Matsushiro seismic swarm based on electro-kinetic effects. In addition, a self-potential (SP) anomaly has been interpreted on basis of the streaming potential model, based on the Onsager relation [17].

Previous descriptions of the electromagnetic field, due to the electro-kinetic effect, have been limited to a stationary case, and the source region is assumed to be at the boundaries of different substances (e. g. [23]). An extended estimation

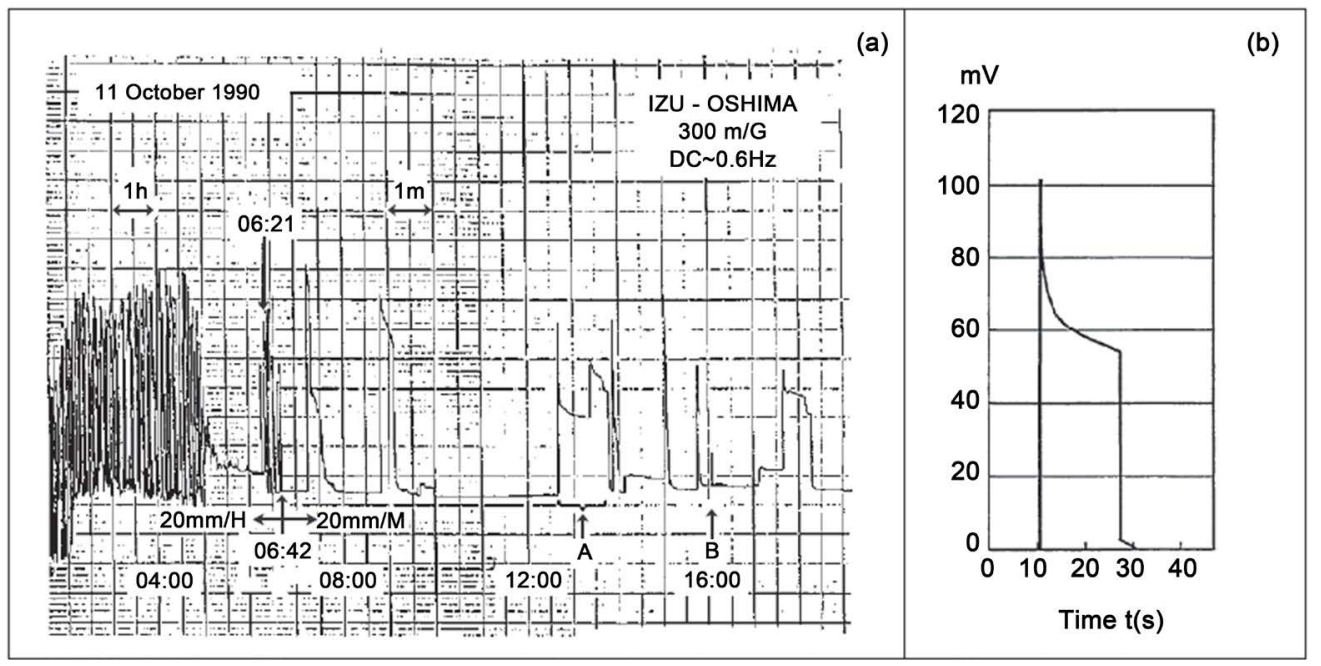

Figure 3. GUV type anomaly. a) the observed waveform. b) simulated waveform assuming the source function. 
of the induced field in the case of transient generating forces was first attempted by [25] using the Maxwell equation. The transient electric field can be estimated if the pressure distributions are given in a specified conductivity distribution. The time evolution of the electrical field is highly similar to that at the zone near the source, because of the diffusion property i.e. the phase 1 and phase 2 of a sharp change are conserved in the near field. However, at several kilometers distance from the source, the signal is sufficiently diffused to lose the original sharpness at the driving point. The property could be also used to infer the source distance.

The analysis of GUV indicates the following points,

1) The electric field anomaly is well correlated with the seismic swarm and volcanic eruptions around the sites.

2) The electric anomaly is suggested to be induced by variations in the confined water circulation regime through electric-kinetic effects.

3) The pore water pressure anomalies, preceding rapid crustal deformation from earthquakes, are suggested to be detected by the borehole antenna.

\section{Co-Seismic EM Field}

\subsection{Co-Rupture Electric Variation}

There are many reports indicating electromagnetic signals are associated with seismic waves, but there is little field observation of waves which propagate faster than P-wave. The first observation was reported using the magnetic measurement [26]. The electromagnetic wave before $\mathrm{P}$ wave arrival, but not starting from the origin time, has been reported frequently [27] [28] [29]. A model based on the piezoelectric effect and the dislocation theory of fault was developed by [27] to explain co-seismic electric signals.

Field detection of the co-rupture electromagnetic anomalies is very rare. One was detected at the time of the great Hokkaido Earthquake in 1994, and the other was the electric field detection starting from the origin time of two moderate earthquakes [30] as presented in this paper.

\subsection{Observed Result}

The anomalies B (Figure 4) observed at Hasaki associated with the major earthquake 4.9 on October 16, 2002 are quite different from the well-known co-seismic electric variation: the co-seismic electric variation has good correlation with the seismic waveform. At this temporary observation we made a continuous high-speed recording of time resolving power of $0.1 \mathrm{~s}$ using the radio time signal adjustment. The recording is shown in the upper part of Figure 4(a). The initial phase of B is clearly seen from $42 \mathrm{~m} 50 \mathrm{~s}$ ( $\mathrm{T}_{0}$ in the Fig.) to $43 \mathrm{~m} 5 \mathrm{~s}$ (P) in the record. $\mathrm{T}_{0}$ is the origin time of the earthquake, determined by the National Research Institute for Earth Science and Disaster Prevention (NIED) [31]. The lower part of Figure 4(b) is the seismic record at Choshi of the strong motion seismograph network (K-NET). 
(a) Electric Filed Change (Hasaki)

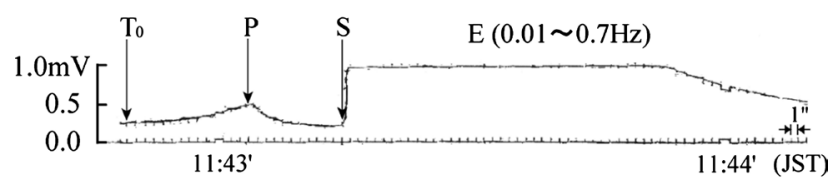

(b) Seismic Record (Choshi)

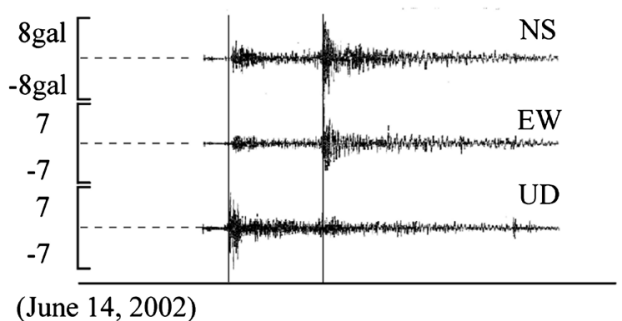

Figure 4. Electric field evolved in good correlation with seismic wave propagation. 1) A high-speed monitoring record is shown. In the recording the time resolution is $1 \mathrm{sec}$ and the clock is calibrated to of $1 \mathrm{~ms}$. $\mathrm{T}_{0}$ shows the origin time of earthquake, and $\mathrm{P}, \mathrm{S}$ are $\mathrm{P}, \mathrm{S}$ waves arrival, respectively at Choshi. The starting time of anomaly is $11 \mathrm{~h} 42 \mathrm{~m} 49.0 \mathrm{~s}( \pm 1$ $\mathrm{s}$ ), very close to the origin time of the earthquake, $\mathrm{T}_{0}=11 \mathrm{~h} 42 \mathrm{~m} 50.0 \mathrm{~s}$. 2) The strong motion record of the earthquake at Choshi near Hasaki (Figure 2). The time axis of the strong motion record is shifted corresponding to the position difference between the electric field observation site HASAKI and the K-net site Choshi.

Comparison of the electric field variation and the observed seismic waveform leads us to the following points,

1) the anomaly started from the origin time of the earthquake,

2) the electric field strength increased gradually until $P$ wave arrival,

3) the strength decreased after P-wave arrival,

4) the strength increased sharply from S-wave arrival, and decreased slowly as the seismic wave diminished,

5) the propagation speed was estimated to be larger than $100 \mathrm{~km} / \mathrm{s}$.

Similar high-speed recordings were taken by Dr. Takahashi for about 27 months, from June 1994 to December 2002: 20 months at Hasaki and 7 months at Chikura. The records were checked by eye to find similar co-seismic signals induced by moderately large earthquakes. Seismic events with a magnitude $M \geq$ 4, and epicentral distance less than $100 \mathrm{~km}$ are selected by use of the official seismic catalog of Japan Meteorological Agency (JMA). Total of 55 earthquakes are picked up. Careful analysis found that there were three cases of co-rupture anomalies in 42 events at Hasaki, and 2 cases in 24 events at Chikura. Those signals were more or less similar, i.e. the biggest signal at S-wave arrival, but the correlation with the seismic wave form is not so clear compared with that of October 16, 2002. Anyhow it is important to know there are another 5 examples of co-seismic electric signals travel with speed larger than $100 \mathrm{~km} / \mathrm{s}$.

\subsection{Mechanism}

The strong correlation of the electric field variation with the P-wave leads us to infer that the generation zone is limited to the area around the seismic wave 
front. [24] indicated that Seismic Electric Signals (SES) are generated, first by active sources such as crustal deformations, crack generation, and pour water movement, second by passive sources such as changes in the physical parameters, i.e. electrical conductivity, magnetic susceptibility and electric permittivity, and third by apparent sources i.e. observation and environment noises. The active sources are of piezo-magnetic, piezo-electric, non-classical piezo-electric, electro-kinetic, and induction effects. The present phenomena do not seem to have been induced by the passive causes because of the large difference of the time constants between the two phenomena. External causes are also discarded because of the high $\mathrm{S} / \mathrm{N}$ ratio of the sensor.

Order estimation of the SES strength [32] suggests that the dominant causes are the seismo-dynamic effect [28] and/or the electro-kinetic effect. The unstable detection of the present phenomena excludes the induction effect. We suspect that the electro-kinetic effect induces an increase in the confined pressure at the wave front [30] [32]. The SES for the case of infinite half space was estimated to indicate that the far field changes as $r^{-2}$ ( $r$. focal distance) [32] and the signal reaches the point up to more than $160 \mathrm{~km}$ from the wave front. These results agree with the present observations.

The phenomenon suggests a new measurement method in seismology using electromagnetic sensors. We can approximate that the first detection time is the origin time, with the result to reduce one of the unknowns in the focal parameters determination. One of most interesting application is for earthquake early warning (EEW). The most developed EEW has been working since 2007 [33] [34] [35] [36] [37]. This system uses the focal parameters determined by P-wave arrival data at a small number of observation sites. The practical use of this information started from October 2007 in Japan with the implementation of counter-measure systems, such as the emergency shutdown of gas fittings and home electrical devices (28). But it generally provides insufficient time (seconds) in cases of shallow depth just under the city. A new electromagnetic sensor will afford additional seconds, along with an accompanying decrease in the extent of earthquake disaster [36] [37].

\section{Microcrack}

The identification of anomalous phenomena is a prerequisite for understanding the physical processes to be applied to earthquake forecasting (e.g., [16] [38]). For short-term prediction, numerous observations by seismic, geodetic, hydrologic and electromagnetic approaches have been conducted to find precursory phenomena. Of them, pre-shocks have been the most direct and plausible phenomenon for identifying the earthquake nucleation stage. Previous investigations for pre-shock activity of natural earthquakes or the micro-cracks activity of laboratory experiments (e.g., [39] [40] [41] [42] [43]) have provided essential evidence for the building of the physical and statistical models of the nucleation process. 
As for natural earthquakes, however, the evidence is limited to an average image over many samples. There is considerable variability in the results with few plausible clues to be applied for practical prediction (e.g., [38]). On the other hand, with the rock fracture experiment we can always observe the characteristic rapid increase of acoustic emission activity in the nucleation period [42] [43], especially in the accelerated increase of micro-cracks known to follow the modified Ohmori formula. However, there are only a few pioneering field studies (e.g., [44] [45] [46]). Those researchers found that the acoustic emission intensity is high during a period spanning about a half-day before and after an earthquake, without means to define the nucleation phase.

\subsection{Results}

An updated data logger was installed at Hasaki on March 3, 2011, 8 days before the Tohoku earthquake. We checked the AC and DC daily records obtained for March 3 to March 31, 2011. A daily record shows nothing except for background noises, seen as a belt on the calmest state and as a short time-period pulse-like activity on active days. All the pulse-like variations were picked up. There is a total of 224 events, including the large event of the co-seismic variation corresponding to the main shock, being consistent with one of the guidelines to approve the candidate of precursor [12].

Whole events are classified into three groups, depending on their signal duration: type A (Figure 5(a)), type B (Figures 5(b)-(d)), and type C (Figure 5(e)). The type A event (Figure 5(a)) is simply a so-called ULF geyser-like variation (GUV). The type B and C events have a much smaller duration, from $10 \mathrm{~ms}$ to several tens of $\mathrm{ms}$ for type B, and 0.3 - $0.5 \mathrm{~ms}$ for type C. Type B pulses are subdivided into three types, depending on their waveform (Figures 5(b)-(d)). Type B-1 (Figure 5(b)) is similar to type A in its abrupt rise and maintaining polarity, similar to the relaxation waveform, but is different in decaying stepwise. Similar electric variation is reported to be associated with acoustic emissions in rock deformation experiments, in a hydraulic fracturing test, and in a simulation to investigate the Loma Prieta Earthquake. Type B-2 (Figure 5(c)) is a wave packet similar to seismic waveform. Type $\mathrm{C}$ (Figure $5(\mathrm{e})$ ) is a wave packet with a carrier frequency of some $5 \mathrm{kHz}$. The crack theory [38] suggests that type A and type B1 correspond to tensile cracks, types B-2 and C to shear modes, and type B-3 to the compounded mode. Figure 6 shows the time evolution of the number of the pulses of types A (green), B (red), C (blue). Just before the great Tohoku Earthquake ("EQ3.11" in the Figure), the type B variations were dominant, and type A rare. After the earthquake, types $\mathrm{A}$ and $\mathrm{C}$ appeared without type $\mathrm{B}$. The three kinds of B band anomalies evolved similarly, but the wave packet type (B-2) tended to be dominant approaching the main shock, in agreement with the rock fracture experiment results.

The time evolution of the type B phenomena had a strong similarity to that of the acoustic emissions just before the rupture in the rock experiment, and to that 
of the pre-shocks before the main shock. Fitting the inverse power law $\left(t_{f}-t\right)^{-n}$ to the rate of occurrence number $N(t)$ of cracks at arbitrary time $t$ (day starting March 1, 2011) from the final rupture time $t_{f}$ results in the index number $n=2.0$ and $t_{f}=10.5$. The index number 2.0 is nearly the same as 1.7 for the foreshock,

(a) DC : A- TYPE

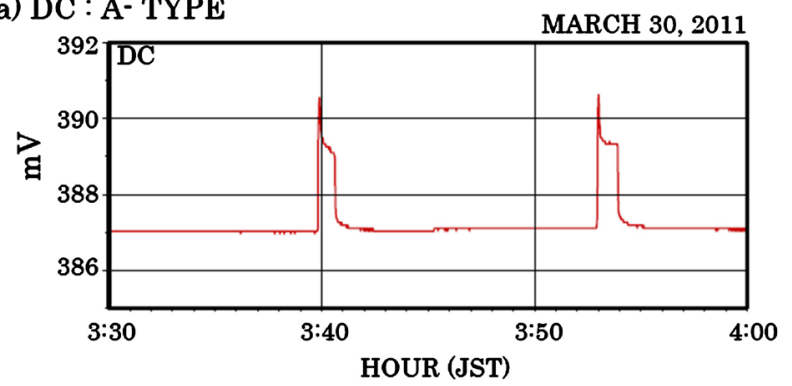

(b) AC : B-1 TYPE

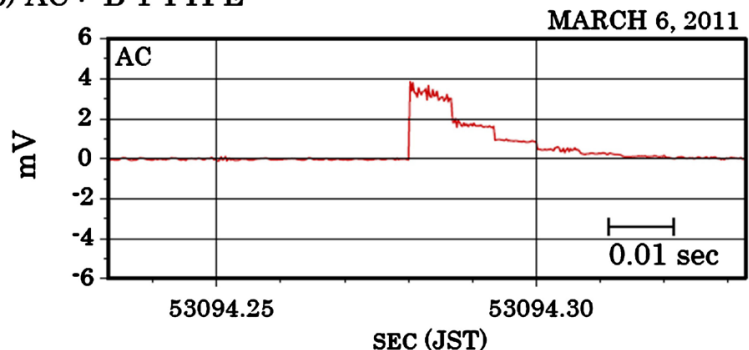

(c) AC : B-2 TYPE

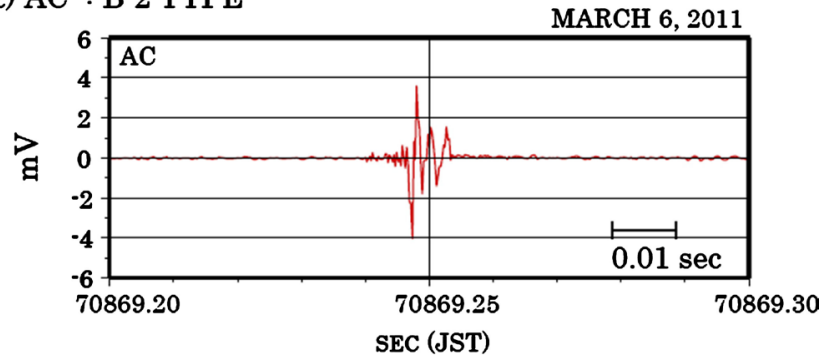

(d) AC : B-3 TYPE

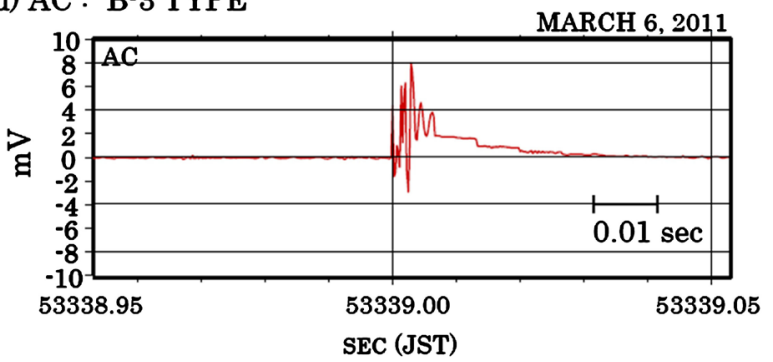

(e) AC : C TYPE

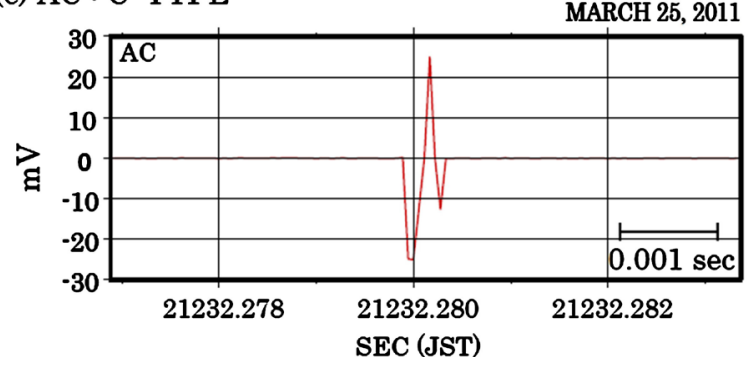

Figure 5. Waveform of the pulse-like signals. 


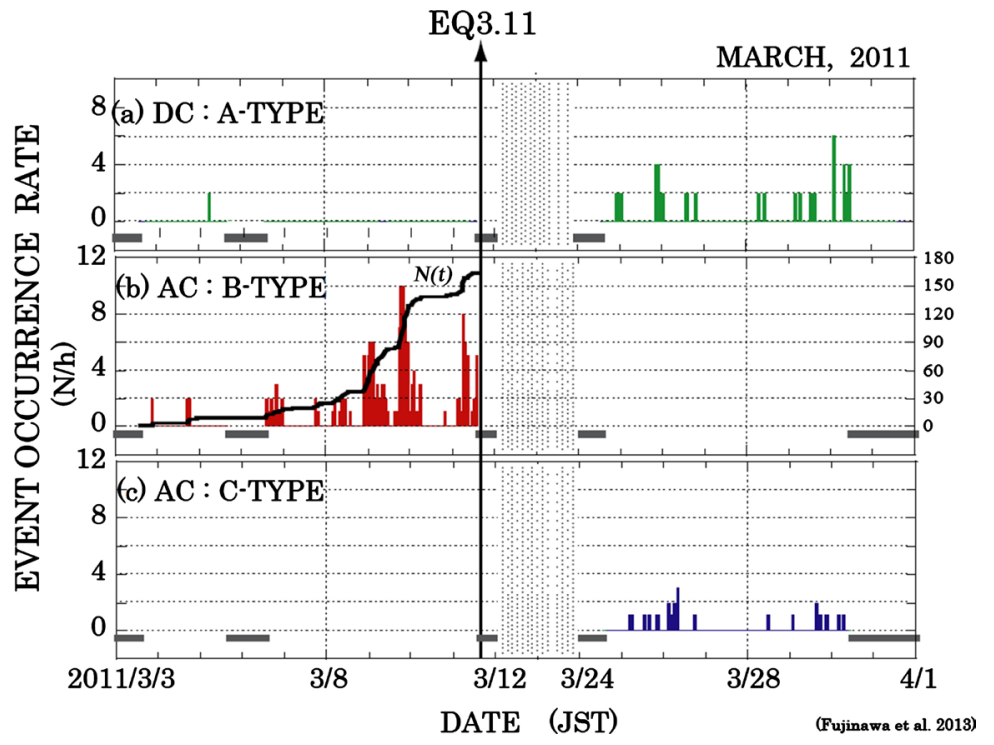

Figure 6. The Tohoku Earthquake, magnitude 9.0 occurred on March 11, 2011. (a) Time evolution pulse-like signal of Type A (green). Almost all of the type A variation occurred after the main shock. (b) Time evolution of Type B (red) including B-1, B-2, and B-3. The type $B$ variation is suggested to be particularly useful for imminent prediction. The continuous curve shows the cumulative number of the whole B-type variations. (c) Time evolution of Type $\mathrm{C}$ (blue). This type occurred only after the after-effect stage of the seismic cycle. The Tohoku Earthquake, magnitude 9.0 occurred on March 11, 2011. There are several time periods (grey horizontal line) without data due to logger problems and malfunction caused by the severe main shock.

but about 4 times larger than $n=0.5$ for the acoustic emission in the laboratory experiment. The assumed time 10.5 agrees satisfactorily with the actual value of 11.6. Enough of many microcracks enabled us to know the time evolution of activity and to suggest the final rupture time.

\subsection{Generation Mechanism (MC)}

The study of seismo-electric variation had a new era after the work of [47]. The physics of wave generation and propagation in porous media, saturated with fluid, was formulated from the microscopic point of view considering the individual motion of main rock substance and the fluid phase. He derived equations for the coupled poro-elastic and EM field on the basis of Biot's poro-elastodynamic equations and from Maxwell's equations of electrodynamics. The wave mode equation for a fixed frequency has been shown to have four modes, fast and slow compressional $\mathrm{P}$-wave $\left(\mathrm{P}_{\mathrm{f}}\right.$ and $\mathrm{Ps}$ ) and two transversal modes ( $\mathrm{S}$-wave and electromagnetic modes: S, EM). The former three modes are co-seismic electric field changes accompanying seismic waves. The EM mode is an electromagnetic wave in the fully saturated porous media with dynamic electric permittivity and dynamic conductivity, depending on the frequency. The analytical form was given by [47] [48]. Those seismo-electric waves have been confirmed in the numerical and laboratory experiments [49]-[55] indicating three phases Pf, S, EM modes are present though the EM mode is extremely small to be observed in the field 
[49] [50] [51].

The electrokinetic coupling model was analyzed by [49] for the double couple sources providing a useful means for multi-disciplinary investigation of earthquake precursor through electromagnetic and seismological approaches. Here the observed anomalous electric field variations are analyzed based on recent results on the investigation of seismo-electromagnetism started by [48].

\subsection{Electric Strength of Each Phases}

The electric field variations associated with microcracks sometimes have a clear P-phase besides a dominant S-phase and some events have a small phase, which was assumed to be EM mode. We made quantitative and qualitative comparisons of the observed and estimated amplitude of each mode using electric field description induced by double couple point source in the isotropic medium [48] [49]. We used the basic formulation of [49] and the subsequent formulation for the double couple source of [49] to compare the observed amplitude with those calculated. [49] showed that the electric field E, induced by double couple of the dipole moment $M_{0}$, is described by the far field, intermediate, and near field parts as per the seismic waves in the elastic medium. In the far field approximation, the electric field Epf of fast $\mathrm{P}$-wave (Pf) and Es of S-wave(S) field are compared [55] for events of shear mode. We used only shear mode events.

The ratio $A p / A s$ is about 30 times: the strength of the Pf-wave is much larger than the $\mathrm{S}$ phase. On the other hand, the observed ratio shows that the strength of the EM mode to that of the $S$ mode is $0.030 \pm 0.02$. The value depends on the wave frequency parameters of the medium. In the case of frequency $300 \mathrm{~Hz}$, the ratio remains almost the same for $A p t A s$, and one third for $A e m / A s$, respectively, compared with those of $500 \mathrm{~Hz}$. The permeability difference of 2 order $(k o=$ $\left.10^{-14} \mathrm{~m}^{2}, k o=10^{-12} \mathrm{~m}^{2}\right)$ results in almost the same values for $A p d A s$, and about a $10 \%$ decrease for $E e m / E s$.

We can see that there is considerable agreement between the observed and estimated electric strength. At the same time, it is noted that $\mathrm{S}$ and EM modes have observed values an order larger than those by estimation; the Pf mode has about $1 / 50$ of the estimated value. The exceptionally large estimated values are discussed in the following.

The electric strength of fast-P, S, and EM modes of the shear type events are compared. The averages of observed amplitude orders as $A s>A p_{f}>A e m$, are partially different from the present and previous numerical simulation results. Analytical estimation is conducted on the basis of the seismo-electromagnetic theory of [47], assuming the isotropic homogeneous medium is filled with confined solvent. The contradictory relation between the observed and numerical simulation results may be attributed to the assumed whole space model rather than the half space model. Overall agreement between the observed and estimated results support that, 1) Pulse-like events of B-type of electrogram before the Tohoku Earthquake in 2011 were induced by the rupture of microcracks in 
the nucleation period of the main shock, 2) Observed phases of waveform, P, S, and EM are confirmed to be co-seismic Pf, $\mathrm{S}$ and free electromagnetic wave assumed by [47], 3) the SES phenomena in the field can be analyzed from the formation of [47] and from successive progress by successors (e.g., Revil).

\section{Conclusions}

We have been observing electric field changes at 13 ground electric field monitoring stations in the central parts of Japan, in relation to the prediction on earthquakes and volcanic eruptions since 1989. The detection sensor is a special antenna made of a vertical pipe casing placed in boreholes. The system is very sensitive to crustal activities, such as earthquakes and volcanic eruptions, and is highly robust to both meteorological and urban noise. Three stages of observation have led to the following results,

1) GUV (Geyser type ULF Variation)

a) The electric field anomaly is well correlated with seismic swarm or volcanic eruptions around sites.

b) It is suggested to be induced by changes of the confined water circulation through electric-kinetic effects.

c) The GUV can be profitable signals to detect precursors of earthquakes and volcanic eruptions.

2) Co-rupture Signal

We presented the observational data showing that there is electromagnetic field variation at the seismic wave front with the effective propagation speed of order $100 \mathrm{~km} / \mathrm{s}$. This phenomenon is suggested to be more useful for EEW technology, producing more lead-time before arrival of the "big shock", when compared to the usual monitoring that relies on elastic wave detection. Further studies are needed before we can develop instruments able to detect electric field variations. Future efforts may need to concentrate on increasing the dynamic range of the measurements as well as gaining understanding of the generation mechanism of the phenomenon.

3) Micro-crack

We have observed that the electromagnetic method can detect microcracks preceding natural earthquakes by use of a special underground antenna. In addition, the detection distance for microcracks is suspected to be much larger than by the acoustic emission method. The long distance detection of microcracks, in comparison to acoustic emissions, is caused by the small decay rate of the seismo-electric signals trapped on the conductivity contrast. The nucleation stage of a crustal rupture can be identified through monitoring of the electric field variations, focusing on particular waveforms in the selected frequency ranges.

These pulse-like phenomena are suggested to be one of the most probable candidates of precursors for an imminent prediction, satisfying many of the conditions required for the precursor by the IAESPI committee. Our present findings could provide a breakthrough for the imminent prediction of earth- 
quakes. This method can also be applied to volcanic eruptions and large-scale ground slides.

4) Remarks

Precursory phenomena are investigated by means of seismic activity, crustal deformation, groundwater anomalies, and electromagnetic anomalies. Present findings of the overall agreement of the observation and estimation for the seismo-elecromagnetic phenomena suggest that seismological, crustal, groundwater, and electromagnetic phenomena that appear in the preparatory process of earthquake occurrence can be analyzed and discussed on the theory of behavior of the seismic activity, hydro dynamical and electromagnetic, and phenomena induced by variety of cracks taking account of the electro kinetic effect. Many of the observational electric anomalies can be more satisfactory interpreted based on the unified scheme. A multidisciplinary approach, based on the unified theory induced by varieties of crack, is expected to open a new window to building up practical earthquake prediction.

\section{Conflicts of Interest}

The authors declare no conflicts of interest regarding the publication of this paper.

\section{References}

[1] Takahashi, T. and Takahashi, H. (1989) Tomography of Seismo-Radio Wave Source Regions for Predicting Imminent Earthquakes. Physics of the Earth and Planetary Interiors, 51, 40-44. https://doi.org/10.1016/0031-9201(89)90211-2

[2] Fujinawa, Y. and Takahashi, K. (1990) Emission of Electromagnetic Radiation Preceding the Ito Seismic Swarm of 1989. Nature, 347, 376-378. https://doi.org/10.1038/347376a0

[3] Fujinawa, Y., Kumagai, T. and Takahashi, K. (1992) A Study of Anomalous Underground Electric Variations Associated with a Volcanic Eruption. Geophysical Research Letters, 19, 9-12. https://doi.org/10.1029/91GL02822

[4] Fujinawa, Y. and Takahashi, K. (1998) Electromagnetic Radiations Associated with Major Earthquakes. Physics of the Earth and Planetary Interiors, 105, 249-259. https://doi.org/10.1016/S0031-9201(97)00117-9

[5] Fujinawa, Y., Takahashi, K., Matsumoto, T., Iitaka, H., Nakayama, T., Sawada, T. and Sakai, H. (2001) Electric Field Variations Related with Seismic Swarms. Bulletin of the Earthquake Research Institute, 76, 391-415.

[6] Fujinawa, Y., Matsumoto, T. and Takahashi, K. (2002) Modeling Confined Pressure Changes Inducing Anomalous Electromagnetic Fields Related with Earthquakes. Journal of Applied Geophysics, 49, 101-110. https://doi.org/10.1016/S0926-9851(01)00102-1

[7] Takahashi, K., Fujinawa, Y., Matsumoto, T., et al. (2000) An Anomalous Electric Field Variation with the Seismic Swarm(1)-Underground Electric Field Observation at Hodaka Station (1995-1999). Technical Notes 204. http://dil-opac.bosai.go.jp/publication/nied tech_note/pdf/KJ-01_204.pdf

[8] Fujinawa, Y., Noda, Y., Takahashi, K., Kobayashi, M., Takamatsu, K. and Natsumeda, J. (2013) Field Detection of Microcracks to Define the Nucleation. Interna- 
tional Journal of Geophysics, 2013, Article ID: 651823. https://doi.org/10.1155/2013/651823

[9] Park, S.K., Johnston, M.J.S., Madden, T.R., Morgan, D.F. and Morrison, H.F. (1993) Electromagnetic Precursors to Earthquakes in the ULF Band: A Review of Observations and Mechanisms. Reviews of Geophysics, 31, 117-132. https://doi.org/10.1029/93RG00820

[10] Hayakawa, M. and Fujinawa, Y. (1994) Electromagnetic Phenomena Related to Earthquake Prediction. Terra Scientific Publishing Company, Tokyo, Japan.

[11] Hayakawa, M. (1999) Atmospheric and Ionospheric Electromagnetic Phenomena Associated with Earthquake. Terra Scientific Publishing Company, Tokyo, Japan.

[12] Wyss, M. (1997) Second Round of Evaluation of Proposed Earthquake Precursors. Pure and Applied Geophysics, 149, 3-16. https://doi.org/10.1007/BF00945158

[13] Fujinawa, Y., Takahashi, K., Matsumoto, T., Iidaka, H., Yamane, S., Nakayama, T., Sawada, T. and Sakai, H. (2000) Electromagnetic Field Anomalyassociated with the 1998 Seismic Swarms in Central Japan. Physics and Chemistry of the Earth $(A), 25$, 247-253. https://doi.org/10.1016/S1464-1895(00)00040-5

[14] Hickman, S., Sibson, R. and Bruhn, R. (1995) Introduction to Special Section: Mechanical Involvement of Fluids in Faulting. Journal of Geophysical Research: Solid Earth, 100, 12831-12840. https://doi.org/10.1029/95JB01121

[15] Wakita, H., Sano, Y. and Mizoue, M. (1987) High 3He Emanation and Seismic Swarms Observed in a Nonvolcanic, Forearc Region. Journal of Geophysical Research: Solid Earth, 92, 12539-12546. https://doi.org/10.1029/JB092iB12p12539

[16] Rikitake, T. (1982) Earthquake Forecasting and Warning. Tokyo Center for Academic Publications, Reidel Publishing, Tokyo, Japan.

[17] Ishido, T. and Muzutani, J. (1981) Experimental and Theoretical Basis of Electrokinetic Phenomena in Rock-Water Systems and Its Applications to Geophysics. Journal of Geophysical Research: Solid Earth, 86, 1763-1775. https://doi.org/10.1029/JB086iB03p01763

[18] Bernard, P. and Le Mouel, J.L. (1996) On Electrotelluric Signals. In: A Critical Review of Van, Earthquake Prediction from Seismic Electrical Signals, World Scientific, New York, 118-152.

[19] Nourbehect, B. (1963) Irreversible Thermodynamic Effects in Inhomogeneous Media and Their Applications in Certain Geoelectric Problems. Ph.D. Thesis, Massachusetts Institute of Technology, Cambridge, MA.

[20] Fenoglio, M.A., Johnston, M.J.S. and Byerlee, D.J. (1995) Magnetic and Electric Fields Associated with Changes in High Pore Pressure in Fault Zones. Application to the Loma Prieta ULF Emissions. Journal of Geophysical Research: Solid Earth, 100, 12951-12958. https://doi.org/10.1029/95JB00076

[21] Majaeva, O., Fujinawa, Y. and Zhitomirsky, M.E. (1997) Modeling of Non-Stationary Electro-Kinetic Effect in a Conductive Crust. J. Geomag. Geoelectri., 49, 1317-1326.

[22] Mizutani, H., Ishido, T., Yokokura, T. and Ohnishi, S. (1976) Electrokinetic Phenomena Associated with Earthquakes. Geophysical Research Letters, 3, 365-368. https://doi.org/10.1029/GL003i007p00365

[23] Fitterman, V. (1978) Electrokinetic and Magnetic Anomalies Associated with Dilatant Regions in a Layered Earth. Journal of Geophysical Research: Solid Earth, 83, 5923-5928. https://doi.org/10.1029/JB083iB12p05923

[24] Gershenzon, N.I., Gokhberg, M.B. and Gul'yel'mi, A.V. (1994) Electromagnetic Field of Seismic Pulses. Physics of the Solid Earth, 29, 789-794. 
[25] Majaeva, O., Fujinawa, Y. and Zhitomirsky, M.E. (1997) Modeling of Non-Stationary Electrokinetic Effect in a Conductive Crust. Journal of Geomagnetism and Geoelectricity, 49, 1317-1326. https://doi.org/10.5636/jgg.49.1317

[26] Belov, S.V., Migunov, N.I. and Sobolev, G.A. (1974) Magnetic Effects Accompanying Strong Kamchatkan Earthquakes. Geomagnetism and Aeronomy, 14, 380-382. (In Russian)

[27] Huang, Q. (2002) One Possible Generation Mechanism of Co-Seismic Electric Signals. Proceedings of the Japan Academy, Series B, 78, 173-178. https://doi.org/10.2183/pjab.78.173

[28] Honkura, Y., Matsushima, M., Oshiman, N., Tuncer, M.K., Baris, S., Ito, A., Iio, Y. and Isikara, A.M. (2002) Small Electric and Magnetic Signals Observed before the Arrival of Seismic Wave. Earth, Planets \& Space, 54, 9-12. https://doi.org/10.1186/BF03352449

[29] Nagao, T., Orihara, Y., Yamaguchi, T., Takahashi, I., Hattori, K., Noda, Y., Sayanagi, K. and Uyeda, S. (2000) Co-Seismic Geoelectric Potential Changes Observed in Japan. Geophysical Research Letters, 27, 1535-1538. https://doi.org/10.1029/1999GL005440

[30] Fujinawa, Y., Takahashi, K., Noda, Y., Iitaka, H. and Yazaki, S. (2011) Remote Detection of the Electric Field Change Induced at the Seismic Wave Front the Start of Fault Rupturing. International Journal of Geophysics, 2011, Article ID: 752193. https://doi.org/10.1155/2011/752193

[31] National Research Institute of Earth Science and Disaster Prevention (NIED) (2011) http://www.bosai.go.jp/

[32] Gershenzon, N.I. and Bambakidis, G. (2001) Modeling of Seismo-Electromagnetic Phenomena. Russian Journal of Earth Sciences, 3, 247-275. https://doi.org/10.2205/2001ES000058

[33] Kanamori, H. (2005) Real-Time Seismology and Earthquake Damage Mitigation. The Annual Review of Earth and Planetary Science, 33, 195-214. https://doi.org/10.1146/annurev.earth.33.092203.122626

[34] Horiuchi, S., Negishi, H., Abe, K., Kamimura, A. and Fujinawa, Y. (2005) An Automatic System for Broadcasting Earthquake Alarms. Bulletin of the Seismological Society of America, 95, 708-718. https://doi.org/10.1785/0120030133

[35] Tsukada, S. and Ohtake, K. (2002) Application of Pattern Recognition to Seismic Event Discrimination, Methods and Application of Signal Processing in Seismic Network Operations. In: Tananami, T. and Kitagawa, G., Eds., Lecture Note in Earth Sciences, Volume 98, Springer, Mercedes-Druck, Berlin, 21-234.

[36] Fujinawa, Y., Kouda, R. and Noda, Y. (2015) The Resilient Smart City (An Proposal). Journal of Disaster Research, 10, 319-325. https://doi.org/10.20965/jdr.2015.p0319

[37] Fujinawa, Y. and Noda, Y. (2013) Japan's Earthquake Early Warning System on 11 March 2011: Performance, Shortcomings, and Changes. Earthquake Spectra, 29, S341-S368. https://doi.org/10.1193/1.4000127

[38] Scholz, C.H. (2002) The Mechanics of Earthquake and Faulting. 2nd Edition, Cambridge University Press, Cambridge, 471. https://doi.org/10.1017/CBO9780511818516

[39] Jones, L.M. and Molnar, P. (1979) Some Characteristics of Foreshocks and Their Possible Relationship to Earthquake Prediction and Premonitory Slip on Faults. Journal of Geophysical Research: Solid Earth, 84, 3596-3608. 
https://doi.org/10.1029/JB084iB07p03596

[40] Ohnaka, M. (1995) A Shear Failure Strength Law of Rock in the Brittle-Plastic Transition Regime. Geophysical Research Letters, 22, 25-28. https://doi.org/10.1029/94GL02791

[41] Maeda, K. (1999) Time Evolution of Immediate Foreshocks Obtained by Stacking Method. Pure and Applied Geophysics, 155, 381-394.

[42] Yoshida, S. and Ogawa, T. (2004) Electromagnetic Emissions from Dry and Wet Granite Associated with Acoustic Emissions. Journal of Geophysical Research: Solid Earth, 109, B09204. https://doi.org/10.1029/2004JB003092

[43] Lei, X., Kusunose, K., Rao, M.V.M.S., Nishizawa, O. and Satoh, T. (2000) Quasi-Static Fault Growth and Cracking in Homogeneous Brittle Rock under Triaxial Compression Using Acoustic Emission Monitoring. Journal of Geophysical Research: Solid Earth, 105, 6127-6139. https://doi.org/10.1029/1999JB900385

[44] Morgunov, V.A., et al. (1991) Geoacoustic Precursor of Spitak Earthquake. Journal of Volcanology and Seismology, 4, 104-107.

[45] Hattori, K. (2003) Results of Acoustic Emission Observation at Matsushiro Station, Japan. International Frontier Research Group on Earthquakes (RIKEN IFREQ) Final Report, Group Director Seiya, UYEDA.

[46] Molchanov, O., et al. (2005) Near-Seismic Effects in ULF Fields and Seismo-Acoustic Emission: Statistics and Explanation. Natural Hazards and Earth System Science, 5, 1-10. https://doi.org/10.5194/nhess-5-1-2005

[47] Pride, S.R. (1994) Governing Equations for the Coupled Electromagnetics and Acoustics of Porous Media. Physical Review B, 50, 15678-15696. https://doi.org/10.1103/PhysRevB.50.15678

[48] Pride, S.R. and Haartsen, M.W. (1996) Electroseimic Wave Properties. The Journal of the Acoustical Society of America, 100, 1301-1315. https://doi.org/10.1121/1.416018

[49] Gao, Y.X. and Hu, H.S. (2010) Seismoelectromagnetic Waves Radiated by a Double Couple Source in a Saturated Porous Medium. Geophysical Journal International, 181, 873-896. https://doi.org/10.1111/j.1365-246X.2010.04526.x

[50] Garambois, S. and Dietrich, M. (2002) Full Waveform Numerical Simulations of Seismoelectromagnetic Wave Conversions in Fluid-Saturated Stratified Porous Media. Journal of Geophysical Research: Solid Earth, 107, 2148. https://doi.org/10.1029/2001JB000316

[51] Araji, A.H., Revil, A., Jardani, A., Minsley, B.J. and Karaoulis, M. (2012) Imaging with Cross-Hole Seismoelectric Tomography. Geophysical Journal International, 188, 1285-1302. https://doi.org/10.1111/j.1365-246X.2011.05325.X

[52] Ren, H.X., Chen, X.F. and Huang, Q.H. (2012) Numerical Simulation of Coseismic Electromagnetic Fields Associated with Seismic Waves Due to Finite Faulting in Porous Media. Geophysical Journal International, 188, 925-944. https://doi.org/10.1111/j.1365-246X.2011.05309.x

[53] Schoemaker, F.C., Grobbe, N., Schakel, M.D., de Ridder, S.A.L., Slob, E.C. and Smeulders, D.M.J. (2012) Experimental Validation of the Electrokinetic Theory and Development of Seismoelectric Interferometry by Cross-Correlation. International Journal of Geophysics, 2012, Article ID 514242. https://doi.org/10.1155/2012/514242

[54] Haas, A.K., Revil, A., Karaoulis, M., Frash, L., Hampton, J., Gutierrez, M. and Mooney, M. (2012) Electric Potential Source Localization Reveals a Borehole Leak 
during Hydraulic Fracturing. Geophysics, 78, D93-D113.

https://doi.org/10.1190/geo2012-0388.1

[55] Fujinawa, Y. and Noda, Y. (2020) Field Observations of the Seismo-Electromagnetic Effect for Monitoring of Imminent Stage of Earthquakes and Volcanic Eruptions. In: Grobbe, N., Revil, A., Zhu, Z. and Slob, E., Eds., Seismoelectric Exploration: Theory, Experiments and Applications, AGU Books. 